Notas y comentarios

\title{
La esfera familiar y la participación económica de las mujeres: una reflexión en torno a la tesis de las preferencias de Catherine Hakim
}

\section{The family sphere and women's economic participation: A reflection on Catherine Hakim's preferences thesis}

Mickens Mathieu*

\section{Resumen}

El objetivo del presente artículo consiste en sintetizar la teoría de las preferencias de Catherine Hakim, que desarrolla una perspectiva bastante original en las reflexiones que se centran en la dicotomía familia y mercado de trabajo en la población de las mujeres. Este análisis busca principalmente exponer, por un lado, las aportaciones de la propuesta de Hakim y sus limitaciones mediante las críticas que ha recibido en los debates sociológicos y demográficos. Por otro, se intenta esclarecer sus aplicaciones en cuanto a las políticas públicas orientadas al mejoramiento de las condiciones de las mujeres en las dos esferas.

Palabras clave: Catherine Hakim; teoría de las preferencias; familia; mercado de trabajo; políticas públicas.

* Universidad Estatal de Haití. Dirección: 10 Imp. Ambroise, Puerto Príncipe, Haití. Correo: mathieumickens05@yahoo.fr ORCID: https://orcid.org/00000001-5520-6477

Nota del autor: Agradezco al doctor Germán Vázquez Sandrín, profesor investigador del Instituto de Ciencias Sociales y Humanidades de la Universidad Autónoma del Estado de Hidalgo, por la revisión del texto. 


\begin{abstract}
The objective of this article is to synthesize Catherine Hakim's theory of preferences, which develops a rather original perspective in the reflections that focus on the dichotomy of the family and the labor market in the female population. This analysis mainly seeks to expose, on the one hand, the contributions of Hakim's proposal and its limitations through the criticisms it has received in sociological and demographic debates. On the other, an attempt is made to clarify its applications in terms of public policies aimed at improving the conditions of women in both spheres.

Keywords: Catherine Hakim; preference theory; family; work market; public policies.
\end{abstract}

\title{
Introducción
}

Las incidencias del ámbito familiar sobre las modalidades de inserción socioprofesional de las mujeres siguen siendo una cuestión de fondo que ha atravesado los debates emergidos de campos diversos de las ciencias sociales. Esta problemática es aún importante en el contexto actual, en donde persisten patrones de distribución segmentada y jerarquizada de la fuerza productiva femenina, a pesar del incremento continuo de su incorporación en el mercado de trabajo. Los ángulos de interpretación y apreciación de la realidad ocupacional de las mujeres son diversos y controversiales.

El propósito del presente trabajo consiste en exponer las contribuciones del corpus analítico-conceptual y metodológico de la tesis de las preferencias de Catherine Hakim en este debate. Aunque no claramente explícita, la autora entiende y modela el concepto de preferencia como la expresión de elección que las mujeres formalizan a partir de las opciones que les ofrecen las esferas familiares y ocupacionales. Según la autora, las preferencias recogen una serie de factores causales, como las actitudes, las fuerzas motivacionales, los estilos de vida y las expectativas, que permiten predecir los comportamientos diferenciados de las mujeres entre los trabajos reproductivos no remunerados y los trabajos productivos remunerados, tomando en cuenta los sistemas de valores y el entorno sociocultu- 
ral concebido como rico, moderno y liberal en el cual se encuentran (Hakim, 2000; 2008).

En lo que se refiere a la priorización del estudio de las mujeres, la tesis de las preferencias de Hakim cobra precisamente su pertinencia en vista de la heterogeneidad y la diversidad de los comportamientos y de las actitudes que caracterizan a esta población en la articulación de sus responsabilidades en las esferas reproductiva y productiva. Dichos comportamientos se diferencian de aquellos hombres, quienes orientan casi sistemáticamente sus preferencias hacia el éxito en los trabajos productivos remunerados y la vida pública. Es oportuno subrayar que estas dos esferas mantienen relaciones bidireccionales muy fuertes, de tal forma que el entendimiento de los comportamientos y los resultados de las mujeres y de los hombres sobre el mercado de trabajo debe tomar en cuenta la incidencia del ámbito familiar. En general, se concibe la unidad familiar como moldeadora de mano de obra que responde a las necesidades del espacio productivo. De igual forma, se la analiza tradicionalmente como un espacio inhibidor para la población de las mujeres y catalizador en la de los hombres, en lo que se refiere a deseabilidad, capacidad, posibilidad de inserción y trayectoria profesional (García y De Oliveira, 1994; Humphries y Rubery, 1994).

Para concretizar el propósito planteado en el presente trabajo, se busca plasmar un dialogo entre el núcleo duro, las contribuciones y las posturas críticas de las grandes líneas de demarcación teórica que se desarrollan en contraposición a la perspectiva analítica de Hakim. El interés del presente análisis se entiende a la luz de la originalidad de las aportaciones de la autora en los estudios sociodemográficos y sociológicos que se desarrollan en torno a la participación económica de las mujeres y a la gestión del espacio privado. La pertinencia de las reflexiones elaboradas en el presente trabajo consiste en presentar las controversias que la perspectiva de Hakim ha traído al campo académico. De forma análoga, este escrito puede ser un insumo de alta validez para estimular debates teóricos y metodológicos tan apasionantes como controvertidos en el contexto latinoamericano y caribeño, donde la tesis de Hakim se encuentra escasamente estudiada y explorada, aun en los centros académicos de notoriedad internacional. 
El texto está dividido en tres partes. En la primera, se expone un panorama general que incluye los fundamentos teóricos de la tesis de las preferencias de Hakim, con objeto de delimitar las contribuciones más pertinentes al entendimiento y la interpretación de los comportamientos de las mujeres en cuanto a las esferas familiares y económicas. En la segunda, se examinan y se dialoga con las diversas contradicciones construidas alrededor de visiones complementarias y alternativas que el enfoque de Hakim ha suscitado en las discusiones académicas. En la tercera parte, se reseñan las aportaciones de la perspectiva de Hakim en las políticas públicas que buscan influenciar de una manera u otra las esferas domésticas y laborales en el esquema de distribución del tiempo de las mujeres.

\section{El núcleo duro de los fundamentos teóricos de la tesis de las preferencias de Hakim}

La teoría de las preferencias es un marco interpretativo bastante original que Catherine Hakim, socióloga británica y profesora investigadora de la London School of Economics, formuló en muchos de sus trabajos (Hakim, 2000; 2002; 2003a; 2003b; 2004; 2006; 2008a; 2008b), específicamente en su libro Work-life style choices in the 21 st century, publicado en el año 2000. De esta teoría se puede destacar la tesis de la autoexclusión o autolimitación de las mujeres sobre el mercado de trabajo. Las premisas fundamentales sobre las cuales se asienta la teoría de Hakim sugieren que la población de las mujeres es heterogénea, diversa y polarizada en cuanto a una serie de características, como las preferencias, los estilos de vida, los intereses, los gustos, el entendimiento de la equidad de género, la gestión y la prioridad del espacio doméstico y profesional. Esta realidad conlleva un mosaico de condiciones de género en lo que se trata de los patrones de elección en la asignación de tiempo dedicado al trabajo remunerado o al trabajo no remunerado. Esta heterogeneidad es fundamental para entender el grado de sensibilidad de las mujeres ante las políticas públicas que plantean objetivos en torno a una o ambas esferas familiares y económicas. 
De forma análoga, los postulados de Hakim suponen que el nivel de inclinación de las mujeres hacia la esfera familiar o la esfera económica puede apreciarse con la elección de la carrera profesional, el nivel de inversión en la adquisición de capital humano y cultural, la capacidad y la disponibilidad a participar sobre el mercado de trabajo, así como las orientaciones laborales y las condiciones de trabajo esperadas en la esfera ocupacional en términos de ingresos y nivel de responsabilidad en la institución en la cual ofrecen sus habilidades productivas.

Basándose en los precedentes postulados, Hakim categoriza y cuantifica los comportamientos dominantes en la población de mujeres ubicadas en las sociedades modernas, liberales, ricas, en materia de prioridad entre las actividades domésticas y económicas. La construcción de los tipos ideales divergentes de Hakim es respaldada por evidencias históricas y empíricas bastante pertinentes obtenidas de encuestas cuantitativas de envergadura nacional conducidas en su mayoría a finales de los años noventa y al inicio de la década 2000 en algunos países europeos, como Gran Bretaña, España, Bélgica, Alemania, República Checa y Suecia (Hakim, 2000; 2002; 2003; 2004).

\section{Primera categoría}

La primera categoría reagrupa a las mujeres centradas sobre la familia (home centered women of full-time homemakers). Cuantitativamente, representan un grupo minoritario (20\% aproximadamente) del conjunto de las mujeres encuestadas por Hakim y se encuentran casi invisibles en los medios y las políticas públicas. Se les reconoce como las féminas que están menos atraídas por las formaciones vocacionales que ofrecen mejores escenarios económicos, por la razón de que otorgan prioridad a los quehaceres domésticos y al cuidado de sus progenituras por encima de los propósitos personales realizables sobre el espacio lucrativo. Su presencia sobre el mercado de trabajo se observa en caso de una importante necesidad económica, para asegurar el sostenimiento del hogar. Hakim argumenta que todas las mujeres pertenecientes a este tipo ideal no presentarían necesariamente una baja disposición o predisposición para acumular un nivel elevado 
de capital humano, puesto que la obtención de cierto nivel de calificación representa un capital cultural que se revela muy importante en el mercado matrimonial, sobre todo para casarse con un esposo con alto grado de calificación. Sin embargo, como se puede entender, su objetivo no es seguir una trayectoria ascendente sobre el mercado de trabajo, en virtud de su propensión a abandonar la carrera profesional después de contraer matrimonio y entrar en la vida adulta.

El grupo de mujeres que centran sus estilos de vida sobre la esfera privada exhiben de forma análoga una baja ambición en materia de compensación salarial (racionalidad no maximizadora). Hay que admitir que, a causa de sus preferencias para el espacio doméstico, no podrían esperar condiciones de trabajo mejores, considerando que el espacio ocupacional está dominado por la racionalidad de las empresas con base en la cual organizan y distribuyen las condiciones de trabajo en función del nivel de rendimiento de los trabajadores.

Las pautas reproductivas y demográficas de este subconjunto femenino son marcadas por una alta propensión a experimentar altos índices de fecundidad y residir en familias numerosas. Se entiende que estas mujeres optan por un modelo familiar tradicional altruista, en donde hay una clara división de los roles y la adscripción de las parejas en las responsabilidades domésticas, y en la aportación de ingresos de acuerdo con el género. De tal manera que el hombre es el proveedor económico único (male breadwinner) y la mujer la administradora de las actividades de reproducción biológica. Hakim demuestra que esta categoría de mujeres es muy sensible a las políticas públicas orientadas hacia la salud y el bienestar familiar. Sin embargo, por ser más proclives a especializarse en los trabajos domésticos, no son receptivas a las intervenciones gubernamentales encaminadas al mejoramiento de la participación y las cualidades laborales de las mujeres.

\section{Segunda categoría}

En la segunda categoría se encuentran las mujeres centradas en la carrera profesional (work-centered women o full-time career centered women), en detrimento de la vida familiar. Como las mujeres que 
tienen una inclinación claramente marcada hacia las actividades del hogar, las mujeres adscritas a esta clase no ocupan la mayor proporción del conjunto de las estudiadas por Hakim. Su peso porcentual gira alrededor de $20 \%$. Esta observación puede parecer sorprendente teniendo en cuenta la nueva cara que ha venido perfilando la mano de obra femenina desde las últimas décadas, como la masificación de su presencia sobre el mercado de trabajo, y la ampliación de su incursión en las profesiones liberales, en los puestos de alta responsabilidad, alta autoridad y alto estatus profesional y gerencial de la jerarquía ocupacional. Todos estos perfiles laborales se han visto influenciados por la implementación de medidas a favor de la igualdad de oportunidades educativas y profesionales entre los géneros, la reestructuración del mercado de trabajo y el incremento de las oportunidades profesionales.

Estando totalmente comprometidas con la esfera productiva, estas mujeres optan por el éxito y la plena inversión en la carrera profesional y las actividades equivalentes, como la adquisición de capital humano, cultural, artístico y político. Proporcionan una importancia primordial a los valores que vehiculan el espacio productivo con fines de optimizar los logros laborales, tales como la alta productividad, la competitividad, el individualismo, el desarrollo y la realización personal. En ruptura con los modelos familiares tradicionales, estas mujeres optan por la independencia o la autonomía económica. Son identificables en su mayoría a partir de su rechazo o su despegamiento de los valores patriarcales, su alta propensión a descuidar (o simplemente renunciar) las responsabilidades domésticas y parentales, a presentar una baja disposición a casarse, ser madres y tener hijos. Con base en estas consideraciones, Hakim sugiere que las mujeres pertenecientes a este tipo ideal son muy sensibles a las políticas públicas diseñadas para aportar algún cambio sobre el mercado de trabajo, así como a la reestructuración de la esfera ocupacional.

\section{Tercera categoría}

La tercera categoría reagrupa a las mujeres denominadas adaptativas (adaptative o ambivalent women). Según los datos recolectados por 
Hakim, son proporcionalmente más numerosas $(60 \%$ aproximadamente) que las dos primeras categorías reunidas. Son las mujeres que tratan de conjugar los valores y las exigencias de la vida activa y la vida familiar. En este caso, no muestran un compromiso permanente o absoluto por ninguna de las esferas. Evitando seguir un patrón de conducta definitivo y buscando sacar la mejor combinación de estos dos mundos, las mujeres adaptativas pueden encontrarse en condiciones de indecisión, de ambivalencia, incluso de conflicto, en lo que se trata de la priorización de la unidad doméstica y la realización de objetivos personales sobre el mercado de trabajo. Estos escenarios de indecisiones pueden incluir otras realidades de igual importancia en la vida de una mujer, como el hecho de tener hijos, el número de descendencia, la duración de las lactancias, la intensidad y el calendario de los nacimientos.

Las mujeres adaptativas pueden desarrollar una serie de estrategias para tratar de compatibilizar las funciones domésticas y las profesionales, tales como la elección de empleos a tiempo parcial, flexibles, temporarios, estacionales, ocasionales, a la demanda, autónomos, a domicilio y teletrabajos. Estos colectivos de mujeres presentan una alta preferencia por las carreras poco sujetas a la obsolescencia técnica (pérdida de habilidades), que les ofrecen puestos de trabajo seguros, les permitan entrar y salir del mercado de trabajo, así como una alta posibilidad de armonizar las exigencias familiares y las profesionales.

Las féminas adaptativas son muy propensas a idealizar modelos igualitarios en lo que se refiere a la organización de la familia y específicamente la equiparación de los roles, la repartición del tiempo y la energía entre el trabajo remunerado y el no remunerado. Estas mujeres pueden tener una fuerte preferencia por la construcción de parejas biactivas o dual-earner (doble proveedores), en lugar de una relación de pareja con roles de género rígidos en materia de distribución de tiempo y sostenimiento económico entre los miembros. Sin embargo, resulta importante subrayar que esta idealización puede no concretizarse en una distribución igualitaria de las responsabilidades de forma rigurosa, sino bastantemente flexible pues las fronteras pueden variar de acuerdo con la familia en donde las mujeres, como sus homólogos hombres, pueden sustituirse en las responsabilidades 
hogareñas y públicas. No hay que minimizar tampoco el hecho de que la realidad puede ser muy diferente de lo que es deseado y de la experiencia vivida en el contexto familiar. La idea subyacente a este planteamiento cobra sentido en la medida que puede existir una brecha importante entre el reparto de las responsabilidades domésticas y la inserción sobre el mercado de trabajo en función del sexo. Este resultado puede ser la expresión tangible de la inadaptación de los roles de género emergente en la construcción de la familia y de la resistencia de los hombres, sin negar la interiorización de una serie de valores genuinos del orden social interconectados con la maternidad y la feminidad (Tobío, 2005; Navarro, 2006).

Hakim ha demostrado que esta categoría de mujeres es muy sensible a las legislaciones, las medidas fiscales, las políticas institucionales que afectan el espacio familiar y el mercado de trabajo, tales como las que ofrecen abanicos de servicios capaces de mejorar tanto el acceso a las oportunidades ocupacionales como la conciliación entre la carrera laboral y las exigencias del espacio reproductivo.

\section{Grafica 1}

Presentación gráfica de las tres clases de mujeres, de acuerdo con la tipología de Hakim

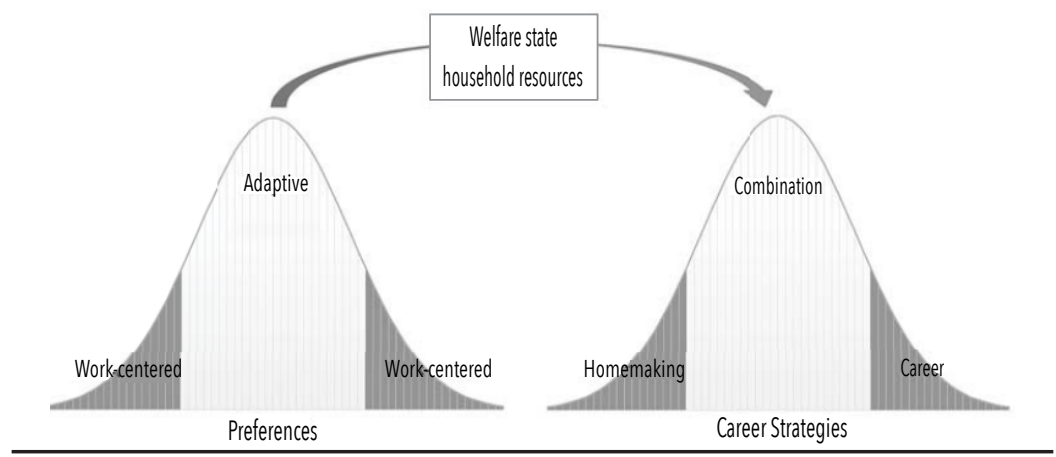

Fuente: Schleutker, 2013. 


\section{Cuadro 1}

Categorización de las tres clases de mujeres según los tipos ideales de Hakim

\begin{tabular}{|c|c|c|}
\hline $\begin{array}{c}\text { Mujeres centradas } \\
\text { en la familia }\end{array}$ & $\begin{array}{c}\text { Mujeres centradas } \\
\text { en la carrera profesional }\end{array}$ & Mujeres adaptativas \\
\hline $\begin{array}{l}\text { Alrededor de } 20 \% \text { de las } \\
\text { encuestadas (puede variar } \\
\text { de } 10 \text { a } 30 \% \text { ). }\end{array}$ & $\begin{array}{l}\text { Alrededor de } 20 \% \text { de las } \\
\text { encuestadas (puede variar } \\
\text { de } 10 \text { a } 30 \% \text { ). }\end{array}$ & $\begin{array}{l}\text { Alrededor de } 60 \% \text { de las } \\
\text { encuestadas (puede variar } \\
\text { de } 40 \text { a } 80 \% \text { ). }\end{array}$ \\
\hline $\begin{array}{l}\text { Comportamientos do- } \\
\text { minados por valores } \\
\text { familiares, cohesión, no } \\
\text { competición y compartir. }\end{array}$ & $\begin{array}{l}\text { Comportamientos do- } \\
\text { minados por valores del } \\
\text { mercado: competitividad, } \\
\text { productividad, individua- } \\
\text { lismo, logros personales, } \\
\text { excelencia, desarrollo } \\
\text { personal. }\end{array}$ & $\begin{array}{l}\text { Comportamientos domi- } \\
\text { nados por compromiso } \\
\text { entre los valores familia- } \\
\text { res y los del espacio pro- } \\
\text { ductivo. } \\
\text { Priorizan modelos fa- } \\
\text { miliares igualitarios en } \\
\text { tareas domésticas y soste- } \\
\text { nimiento económico. } \\
\text { Trayectoria profesional } \\
\text { a menudo truncada o en } \\
\text { espera por nacimiento y } \\
\text { crianza de niños. }\end{array}$ \\
\hline $\begin{array}{l}\text { Dan prioridad a la vida } \\
\text { familiar y crianza de los } \\
\text { niños. } \\
\text { Tienen alto índice de } \\
\text { fecundidad y familias nu- } \\
\text { merosas. } \\
\text { No tienen fuerte apego } \\
\text { al mercado de trabajo. } \\
\text { En general, prefieren no } \\
\text { trabajar. }\end{array}$ & $\begin{array}{l}\text { Dan prioridad a la vida } \\
\text { laboral y pública. } \\
\text { En su mayoría, prefieren } \\
\text { no tener hijos. }\end{array}$ & $\begin{array}{l}\text { Tratan de combinar vida } \\
\text { profesional y vida laboral. } \\
\text { Pueden no tener plan de } \\
\text { carrera fijo. }\end{array}$ \\
\hline $\begin{array}{l}\text { Poca inversión en adqui- } \\
\text { sición de capital humano. } \\
\text { El estudio de una carrera } \\
\text { se concibe más como } \\
\text { acumulación de capital } \\
\text { cultural que como deter- } \\
\text { minante de inserción so- } \\
\text { bre el mercado de trabajo. }\end{array}$ & $\begin{array}{l}\text { Larga inversión en adqui- } \\
\text { sición de capital humano, } \\
\text { entendido como factor } \\
\text { central de inserción y } \\
\text { ascensión sobre el mer- } \\
\text { cado de trabajo y la vida } \\
\text { pública. }\end{array}$ & $\begin{array}{l}\text { Estudian una carrera con } \\
\text { el objetivo de trabajar. } \\
\text { Elección de carreras con } \\
\text { baja obsolescencia técni- } \\
\text { ca y que facilitan la con- } \\
\text { ciliación entre las esferas } \\
\text { productiva y reproduc- } \\
\text { tiva. }\end{array}$ \\
\hline
\end{tabular}

(continúa) 


\section{Cuadro 1}

(concluye)

\begin{tabular}{|c|c|c|}
\hline $\begin{array}{c}\text { Mujeres centradas } \\
\text { en la familia }\end{array}$ & $\begin{array}{c}\text { Mujeres centradas } \\
\text { en la carrera profesional }\end{array}$ & Mujeres adaptativas \\
\hline $\begin{array}{l}\text { Muy responsivas a las po- } \\
\text { líticas sociales orientadas } \\
\text { hacia el bienestar de las } \\
\text { familias. } \\
\text { No receptivas a las polí- } \\
\text { ticas públicas que buscan } \\
\text { aumentar su participación } \\
\text { en la esfera productiva. }\end{array}$ & $\begin{array}{l}\text { Muy receptivas a las po- } \\
\text { líticas públicas buscando } \\
\text { mejorar las oportunidades } \\
\text { económicas, artísticas y } \\
\text { culturales para ellas y la } \\
\text { sociedad en general. }\end{array}$ & $\begin{array}{l}\text { Muy sensibles a políticas } \\
\text { públicas de igualdad de } \\
\text { oportunidades entre mu- } \\
\text { jeres y hombres sobre el } \\
\text { mercado de trabajo: ser- } \\
\text { vicios de cuidado infantil, } \\
\text { trabajo a tiempo parcial, } \\
\text { trabajo flexible. } \\
\text { Sensibles a ciclos econó- } \\
\text { micos, recesión y creci- } \\
\text { miento económico. }\end{array}$ \\
\hline
\end{tabular}

Fuente: Elaboración propia con base en Hakim, C. (2000), Work-lifestyle choices in the 21 st century. Oxford: University Press.

Para terminar esta sección, parece relevante plantear algunos puntos de convergencia y de divergencia entre las tesis de Becker y de Hakim. En efecto, Hakim con su "teoría de las preferencias" concuerda con Becker $(1965 ; 1987)$ con su "nueva economía de la familia" en lo que concierne a la importancia del nivel de capital humano acumulado en la orientación de las preferencias, la división del trabajo y las estrategias de conciliación priorizadas por las mujeres entre las responsabilidades de la unidad doméstica y de la esfera productiva. Los dos autores predicen una tasa de participación o de dedicación más elevada sobre el mercado de trabajo en las mujeres con dotación más elevada de capital humano.

De acuerdo con las conclusiones de Becker (específicamente), el comportamiento de las mujeres altamente educadas se explica por su costo de oportunidad, aproximado por la tasa de utilización (o de depreciación) de los conocimientos acumulados, el nivel de percepción salarial o de rentabilidad de las inversiones en formación profesional, que se encuentra muy elevado; de tal manera que la deserción del espacio productivo para dedicarse a las actividades de cuidado y de crianza en la esfera doméstica parecería una decisión irracional, en vista de que estas mujeres renunciarían a un nivel de ingreso relativamente importante. Además, el salario que percibirían sobre el mercado 
de trabajo les permitiría compensar el costo de los trabajos domésticos no realizados mediante el apoyo pagado de una persona externa.

La lógica inversa es también aplicable para la población de mujeres con baja acumulación en capital humano. Cabe notar también que, según la perspectiva de Becker -fundamentada en la complementariedad, las ventajas comparativas de los cónyuges, la división sexual del trabajo y la especialización de las mujeres en las actividades domésticas-, es una decisión racional y eficiente en la medida que permite maximizar la producción de bienes y servicios necesarios para el bienestar de la unidad doméstica.

Hay que subrayar también el hecho de que estas dos perspectivas son en el fondo ciegas al género de los cónyuges. En efecto, para Hakim, la población que tiene el perfil productivo más homogéneo es la que tiene el resultado más importante sobre el mercado de trabajo. Y para Becker, el colectivo que cumple con el nivel de productividad, de ventaja comparativa más elevado para las actividades productivas, es el que tiene el rendimiento más elevado sobre el ámbito laboral, y el que debería especializarse en las actividades de este ámbito. El inverso de estas dos proposiciones es también válido, tomando en cuenta la unidad doméstica y el campo profesional, y los colectivos de mujeres y hombres.

Cabe resaltar que Hakim, al igual que Becker, consideran a la familia como un espacio de consenso, de intereses convergentes y, de esta forma, hacen abstracción de posibles relaciones de poder, problemas, tensiones, conflictos e intereses no fácilmente armonizables dentro del espacio doméstico. De la misma forma, los dos autores minimizan la influencia de los condicionantes estructurales y culturales en las elecciones que realizan las mujeres en lo que se refiere al hogar y al mercado de trabajo. Asimismo, la racionalidad individualizada y estandarizada representa uno de los razonamientos fundamentales sobre los cuales se asientan las tesis de Hakim y de Becker, sobre todo en lo que concierne a la dedicación entre los trabajos domésticos y los extradomésticos, y también a la producción, la gestión y la distribución de los recursos entre los miembros de la unidad doméstica (Sánchez Mira, 2016).

En cambio, Hakim no concuerda con Becker $(1965 ; 1987)$ en lo referente a la homogeneización del espacio productivo. Del mismo 
modo, la perspectiva de Hakim proporciona informaciones que ayudan a diseñar e implementar políticas públicas mejor dirigidas hacia grupos específicos de mujeres, al contrario de la de Becker. De la misma forma, Hakim construye su tesis partiendo de evidencias empíricas recolectadas sobre el terreno, mientras Becker parte de las hipótesis y de las deducciones convencionales y ortodoxas del campo económico neoclásico, como la maximización de la satisfacción y la utilización óptima de los recursos.

\section{Relevancia de la tesis de Hakim}

De acuerdo con los puntos de vista de la autora, las premisas de la tesis de las preferencias (así como de las explicaciones centradas en la autoexclusión o autocensura) adquieren relevancias irrefutables desde los últimos decenios a causa de una serie de transformaciones sociohistóricas fundamentales que han ido abriendo mayores oportunidades a las mujeres en términos de adquisición de capital humano y participación en el mercado de trabajo. Con base en las amplias elecciones y oportunidades genuinas que están a su alcance, Hakim revindica la posición de que las mujeres están viviendo un momento único en la historia de la humanidad, en donde se encuentran libres de tomar decisiones acerca de sus proyectos de vida en función de sus preferencias personales.

Estas transformaciones se articulan con: a) la revolución y la democratización de los métodos anticonceptivos, que han proporcionado a las mujeres, sobre todo a las sexualmente activas, una posibilidad sin precedente de controlar su sexualidad y planificar su fecundidad y su maternidad; b) las políticas públicas y los mecanismos institucionales que combaten la discriminación y promueven el derecho a la diferencia y a la igualdad de las oportunidades; estas medidas han garantizado a las mujeres un acceso sin precedente a la formación profesional y a puestos de trabajo en todas las posiciones y en todos los sectores del mercado laboral; c) la terciarización de la estructura ocupacional y la expansión de las ocupaciones de cuello blanco (white collar), que atraen en mayor medida a la mano de obra femenina, al contrario de los empleos de cuello azul; d) la flexibi- 
lización del mercado de trabajo por la creación de puestos a tiempo parcial, que son muy atractivos para las personas que no quieren priorizar el espacio laboral sobre las responsabilidades domésticas; e) la expansión de los valores de libertad (elección, decisión en consonancia con el uso de tiempo, y esfuerzos personales) en las sociedades modernas y liberales que otorgan a las mujeres márgenes de maniobra importantes en cuanto a los estilos de vida (hoy en día, en dichas sociedades no existen certezas, convenciones, valores fijos universalmente aceptados sobre los estilos de vida; al contrario de lo que se observa en las comunidades llamadas tradicionales); y $f$ ) las políticas fiscales que disminuyen las cargas tributarias de las familias que tienen hijos.

De acuerdo con Hakim, estos detonantes son esenciales para entender que las causas de los patrones segmentado y estratificado que caracterizan la realidad de los grupos de mujeres entre sí y entre sus pares masculinos, son esencialmente personales, en términos de dotación en capital humano, elección de carrera profesional y sector de inserción sobre el mercado ocupacional. Siguiendo esta misma línea de ideas, invita a los analistas a dar muestras de prudencia en el momento de interpretar las brechas de cualidades laborales (y sus corolarios: segregación horizontal, segregación vertical, piso pegajoso, techo de cristal, rareza en los puestos ejecutivos o en la cima de la jerarquía ocupacional) que caracterizan los resultados de las mujeres sobre el mercado de trabajo, con el fin de evitar explicaciones y predicciones que se fundamentan exclusivamente sobre el patriarcado, los estereotipos y la discriminación de género, tanto de las familias en términos de acumulación de habilidades productivas antes de entrar al mercado de trabajo, como de las empresas en el momento de contratar la mano de obra femenina.

Como se ha descrito en los grupos de mujeres que idealizan la gestión de la esfera familiar, así como las que buscan aprovechar lo mejor de los ámbitos familiares y profesionales, el patrón de inserción de las mujeres en ciertas categorías de empleos (a veces atípicos, flexibles, inestables, de baja calidad y de baja remuneración) puede ser la consecuencia de una libre y legítima elección que se realiza en función de su cosmovisión, idiosincrasia y objetivos de vida. Como se vio anteriormente, Hakim plantea que su teoría es también 
aplicable para predecir otros comportamientos de igual importancia en la vida femenina, como la fecundidad y la nupcialidad.

\section{Diálogo entre la tesis de las preferencias de Hakim y sus críticos}

La perspectiva de las preferencias de Hakim ha sido objeto de debates analíticos fructíferos, controversiales, a veces amargos, en los campos de la sociología y la demografía. Estas discusiones han contribuido a la apertura de nuevas líneas de investigación y a la construcción de novedosos matices explicativos y comprensivos en torno a la esfera familiar y la participación económica de las mujeres.

Como es el caso de la mayoría de los marcos teóricos de tipo individualista o voluntarista, las críticas más pertinentes a la propuesta de Hakim se concentran en la poca atención que la autora acuerda con los sesgos androcéntricos, los determinismos normativos, el entorno sociocultural o marco estructural (con sus restricciones y sus facilitaciones, sus ventajas y sus desventajas), incluso con la biografía personal contextualmente situada en el proceso de formación de las preferencias individuales, en la elección de una carrera profesional y un tipo de trabajo, en el acceso a las oportunidades productivas $\mathrm{y}$ en la progresión diferencial de las mujeres sobre el mercado de trabajo.

Viendo la huella de la estructura económica, de las instituciones y de la cultura en la conformación de los comportamientos reproductivos y laborales de las mujeres, las críticas (en su mayoría feministas) a la tesis de las preferencias describen una cadena de causalidad directa e indirecta que limita a las mujeres en el momento de elegir las opciones que les ofrecen los espacios familiares y laborales. Estos ejes de explicación y predicción son también llamados para analizar y anticipar comportamientos conexos vinculados con la violencia, o la dedicación a los trabajos de cuidado de personas dependientes. Los condicionantes estructurales pueden igualmente contemplar el peso (limitador o facilitador) de algunos factores materiales (tradicionales, liberales) en la actitud de las mujeres en cuanto a la adaptación y la satisfacción por la vida familiar y laboral, como el régimen de bienestar, las políticas familiares, la disponibilidad y la accesi- 
bilidad a medios de transporte, y la posibilidad de contar con apoyos internos o externos (pagados) (Ginn et al., 1996; Philips, 2004; McRae, 2003; Doughney y Leahy, 2006; Tomlinson, 2006; Kangas y Rostgaard, 2007; ManYee, 2007; Perrons et al., 2007; Gash, 2008).

De acuerdo con las tesis feministas, el gran pecado de Hakim se vincula igualmente con el hecho de considerar que las preferencias de estilo de vida y/o la autolimitación de ciertos grupos de mujeres sobre el mercado de trabajo surgen de la nada, o se realizan de forma libre y sin restricciones. De la misma forma, critican la tesis de Hakim por tener un enfoque neotradicionalista de género, por no tomarse la pena de explicar el origen de los comportamientos diferenciados entre las mujeres y los hombres en lo que se refiere a la inserción sobre el mercado de trabajo y a la participación en los oficios vinculados con los cuidados y la reproducción doméstica. De forma análoga, el trasfondo de las consideraciones feministas invita a preguntarse por qué las mujeres son las que tenderían a autolimitarse profesionalmente, en comparación con los hombres. En efecto, según sus puntos de vista, las elecciones que realizan las mujeres están íntimamente vinculadas con el aprendizaje, la interiorización (asimilación) y la reproducción de referencias normativas, valores, creencias, estereotipos y prejuicios sexistas (observables e inobservables) que moldean los comportamientos y programan la identidad social de las mujeres desde la primera infancia. Estas normas y creencias se encuentran en la base de las relaciones intergenéricas de poder, y en la distribución de privilegios, estatus y prestigio en los espacios privados y públicos. De forma concreta, se encuentran vinculadas con la división sexual de las tareas domésticas no remuneradas y extradomésticas remuneradas; con la desigualdad sexual en la generación y distribución de las riquezas, la acumulación de capital humano, la disponibilidad y la capacidad de captar las oportunidades, y seguir una trayectoria ascendente sobre el mercado de trabajo.

Hasta en los contextos actuales, las mujeres son analizadas tanto en los espacios privado-domésticos como en la esfera productiva a partir de sus roles tradicionales de madres, esposas, amas de casas, administradoras de las actividades arraigadas a la maternidad, el cuidado, la subsistencia, la reproducción biológica y material cotidiana (crianza de niños, cuidado de personas mayores, curación de 
personas enfermas). Pues, al fin y al cabo, siguen asumiendo dobles jornadas laborales, segregadas en ocupaciones precarias, repetitivas y menos valorizadas económicamente en las jerarquías ocupacionales. Su trayectoria laboral es generalmente interrumpida en los momentos de embarazo, nacimiento y crianza de los niños. Estas consideraciones se evidencian en la representación gráfica de la trayectoria laboral de las mujeres, que se presenta tradicionalmente en forma de $\mathrm{M}$ más o menos pronunciada según los grupos de mujeres considerados, marcada por el incremento de la participación en el debut de su carrera, el retraso sobre el espacio productivo durante el momento de crianza de los niños, y en seguida por el aumento de su vida activa.

Las preferencias de las mujeres son también influenciadas por las políticas de las empresas, los procesos discriminatorios institucionales, la disparidad de poder y de recursos, y también de capacidad de negociación de los grupos sociales de acuerdo con los contextos socioeconómicos y culturales en los cuales se encuentran. Según los argumentos feministas, los condicionantes externos no hacen más que reducir las posibilidades, levantar barreras, excluir, segmentar con base en el sexo las opciones laborales, las condiciones de trabajo y los privilegios, los cuales, al final de cuentas conducen a limitar el nivel de compromiso de las mujeres sobre el mercado de trabajo (Ginn et al., 1996; McRae, 2003; Crompton Brockmann y Lyonette, 2005, Doughney y Leahy, 2006; ManYee, 2007; Gash, 2008).

Sin negar el deseo de mostrar la heterogeneidad de la población femenina, autores como Duncan (2006) piensan que el nivel triple de desagregación sobre el cual se basa el marco analítico de Hakim es aún incompleto, demasiado reductible a la luz de la complejidad de la realidad sustentada por la socioconstrucción de los roles de género tradicionales, las preferencias, y las elecciones que caracterizan la vida hogareña y laboral de las mujeres en distintos contextos y acontecimientos sociales. Estos autores piensan que los fundamentos teóricos de Hakim esquivan de forma análoga la diversidad de los contextos socioculturales e institucionales (tradiciones, valores, normas) que se encuentran arraigados destacadamente en los diferentes modelos de comportamientos reproductivos y productivos de la población de mujeres. 
Algunos analistas (Cartwright, 2004; Himmelweit y Sigala, 2004; Walters, 2005; Philp y Wheatley, 2010) subrayan el carácter determinista y estático de la propuesta de Hakim, entendiéndola a partir del poco énfasis que la autora concede al carácter repetido de la dinámica de la toma de decisiones y de la construcción de las preferencias de las mujeres, que se ha ido modificando y adaptando a lo largo de su curso de vida en función de las transformaciones acontecidas en el seno de la familia y en el mercado de trabajo. En efecto, la presencia de las mujeres en los puestos de trabajo tradicionalmente reservados a la mano de obra masculina es ahora más frecuente y más importante.

Duncan e Irwin (2004) van en el mismo sentido, mostrando que las preferencias ante lo doméstico y lo productivo se alimentan con procesos originados en los sistemas morales socialmente aceptados y negociados, otorgándoles caracteres cambiantes con el espacio y el tiempo. Estos mismos autores ponen también de manifiesto el poco énfasis que hace Hakim a la noción de clase social, espacio de residencia y regímenes de bienestar en la construcción de su argumentación sobre la configuración de los comportamientos reproductivos y productivos de las mujeres. En efecto, según los resultados de sus investigaciones, la valoración y el significado otorgados a las actividades domésticas y al cuidado de los hombres -la autoridad masculina en el hogar matrimonial-, son diferentes entre las clases populares y las clases medias o superiores. La negociación de los trabajos hogareños, así como los grados de compromisos asumidos por los hombres en las tareas domésticas y de cuidado, es más elevada a medida que se asciende en las clases sociales. Esta observación sugiere la emergencia de cierta metamorfosis de la masculinidad y de la paternidad, sobre todo en los estratos más elevados de la sociedad. Por lo tanto, el modelo normativo patriarcal basado en lo masculino como figura de autoridad y de sostén familiar parece ser más fuerte en las clases populares. Analizando sus puntos de vista, se entiende la relevancia de fomentar el empoderamiento de las mujeres y las actividades de concientización, visibilización y seguimiento de los resultados y los desafíos no solamente en el espacio productivo, sino también en las actividades reproductivas no comerciales que se reconocen como fundamentales para sostener la vida, el bienestar humano y la organización del sistema socioeconómico. 
En contraposición con las reflexiones anteriores, Hakim sostiene que no niega la influencia inhibitoria de la macroestructura sociocultural; sin embargo, no la incluye en su propuesta analítica porque destaca que es marginal en la construcción de las preferencias reproductivas y productivas de las mujeres. De forma análoga, la autora plantea que el hecho de poner un foco desmedido tanto sobre los aspectos exógenos e institucionales, como sobre los arreglos de género tradicionales, puede ser concebido como una posición analítica y metodológica imprudente. En algunos de sus trabajos, Hakim (1995; 2011) llega a etiquetar estos enfoques interpretativos de mitos feministas, en la medida que menosprecian el libre albedrío, las aspiraciones y las prioridades profesionales y matrimoniales, así como la posibilidad de elección autónoma legítima de acuerdo con un proceso de ordenamiento y jerarquización de las alternativas que, en materia de bienestar y de satisfacción de sus necesidades, los escenarios laborales y familiares ofrecen a las mujeres. Estas alternativas no deben ser concebidas como mutuamente excluyentes, en la medida que pueden ser equivalentes o mantener relaciones de indiferencia.

De forma análoga, es relevante plantear que el hecho de subestimar las múltiples relaciones de interdependencia (tipo pushpull) entre las agencias individuales y la macroestructura es un riesgo analítico, puesto que la formación de las preferencias, la dirección y el mantenimiento de las acciones derivadas hacia un ámbito elegido puede ser resultado de un largo proceso de interacción bastante complejo entre las esferas macroestructural, familiar e individual. De ahí viene un sistema autosostenido que comprende los impulsos intrínsecos, los imaginarios y las idiosincrasias. Esta lógica se justifica también por la voluntad de realización y satisfacción de las expectativas de vida en función de las aspiraciones personales. Tal razonamiento cobra sentido en lo concerniente a los significados que las personas otorgan tanto a sus comportamientos, como a los resultados obtenidos (en términos de recompensas objetivas y subjetivas, rendimientos, premios, amenazas o castigos) por haber priorizado tal o cual forma elegida entre la dicotomía familiar y laboral.

En esta misma línea de pensamiento surgen los cuestionamientos que se fundamentan en la obligatoriedad del trabajo extradoméstico de las mujeres y la dedicación plena al trabajo extradoméstico como 
forma de autorrealización de vida. De esta forma, es posible hacerse la siguiente pregunta: ¿las mujeres se insertan sobre el mercado de trabajo porque quieren liberarse del supuesto yugo doméstico o porque no tienen otra opción? La cuestión de la obligatoriedad de insertarse sobre el mercado de trabajo tiene también sentido a raíz de los problemas económicos que enfrentan las familias, en donde un solo sueldo resulta, en la mayoría de los casos, insuficiente para la manutención y el bienestar económico de la familia. En este caso, las familias tienen pocas opciones, entre las que figuran la decisión de enviar a sus miembros, incluso a las mujeres y a los hijos de menor edad, a la esfera productiva a fin de generar los recursos económicos esenciales para su sostenimiento.

Existe toda una línea de investigación que se desarrolla sobre la centralidad (exclusiva) y localización hegemónica del empleo, el salario y el mercado como espacios del orden social que se convierten en indispensables tanto para la supervivencia de los hogares y las familias y la organización de la vida cotidiana, como para la adquisición de prerrogativas, privilegios, estatus y movilidad social. Estos planteamientos encuentran eco en los trabajos de autores como Castel (1994; 1995; 2009; 2010), Prieto (2000; 2007) y Meda (1995), quienes plasman la idea de que el mercado de trabajo ha adquirido importancia desmedida, de tal manera que se convierte en el epicentro, la matriz de la socialización (o de identidad socializadora), de la movilización de las redes sociales que determina el destino vital de las personas, incluyendo a las mujeres, hasta tal punto que nadie puede esconderse o escaparse de él.

Siguiendo los aportes de estos autores, este tema ofrecería la oportunidad para realizar un balance crítico sobre los impactos reales de la centralidad y la sobrelegitimación del mercado de trabajo remunerado en las condiciones de existencia individual y colectiva, tomando en cuenta que ha traído a la masa popular preocupaciones conectadas con las crisis o la destrucción de los regímenes de bienestar, la privatización de los servicios públicos, las incertidumbres, las vulnerabilidades (retos y riesgos), la precariedad, la pauperización, la exclusión (o desafiliación, según los términos de Robert Castel), el desempleo, las desigualdades, la estratificación, la jerarquización, la fragmentación, la segregación (horizontal y vertical), la marginali- 
dad, la subordinación, la desprotección, la inseguridad, la atipicidad, la punibilidad, la estigmatización, la inestabilidad, la explotación, la frustración, la pobreza, la miseria, la carencia, la desesperación, la limitación y la restricción. La lista no es exhaustiva.

De la misma forma, la dedicación de forma consciente al trabajo doméstico y familiar puede ser concebida como una forma de autorrealización, un estilo de vida que los colectivos de mujeres pueden elegir en toda libertad y de forma legítima con base en los valores que definen su personalidad y su identidad. Este planteamiento sugiere que el estudio de una carrera larga, la dedicación completa al mercado de trabajo, el sacrificio del espacio familiar y de los vínculos afectivos para alcanzar el éxito profesional, no debe ser la única forma de realizarse en la vida, alcanzar la emancipación y la felicidad. De hecho, el mercado de trabajo recoge una serie de características interconectadas con la inequidad y la vulnerabilidad, hasta tal punto que sólo una baja proporción de mujeres llega a alcanzar una condición laboral realmente atractiva en términos de puestos de responsabilidad, percepción salarial, acceso a seguro médico y contratos estables. Estas observaciones dan lugar a preguntarse por qué se acepta vivir en un mundo donde es obligatorio estudiar durante muchos años para esperar alcanzar un nivel básico de bienestar en términos de rentas, construcción de un patrimonio, acceso a la salud, a la formación y a la cultura de forma general.

Siguiendo esta misma idea, se entiende que una mujer puede también rechazar la idea de ser una superwoman capaz de enfrentar la duplicidad de la presencia career-care o cosechar éxito en los ámbitos profesional y familiar, tomando en cuenta lo que tienen de exigencia, obligación, competitividad, sobrecarga de trabajo físico y cansancio emocional. De forma análoga, la dedicación de forma completa de las mujeres al trabajo del hogar no está necesariamente vinculada a la frustración y la explotación. No quiere decir que las mujeres son víctimas sin defensa del sistema patriarcal, de los hombres o de la sociedad en general (Hakim, 2011). 


\section{La tesis de las preferencias ante las políticas públicas orientadas hacia las mujeres}

La perspectiva de Hakim ha despertado debates académicos contradictorios entre los científicos sociales y ha adquirido sumo interés por parte de las políticas públicas orientadas hacia los estilos de vida y las opciones de las mujeres ante las responsabilidades familiares y laborales. Por lo tanto, sus reflexiones parecen tener una resonancia más fuerte en las políticas públicas familiares de los países europeos (Gran Bretaña, España, Holanda) y Oceanía (Australia) (Cartwright, 2004; Yerkes, 2013), que en los países latinoamericanos y caribeños.

Para abrir un corto paréntesis, hay que decir que es muy difícil plantear una explicación sólida en lo que concierne a la falta de notoriedad y utilización de los instrumentos analíticos propuestos por la tesis de Hakim en la región latinoamericana y caribeña. Las revisiones conducidas por García y De Oliveira (1994; 2007), García y Rojas (2002), De Oliveira y Ariza (1999), González de la Rocha (1999), Arriagada (2001a; 2001b) y Acosta (2003) sobre las temáticas de familia y trabajo, y de familia y género en la región latinoamericana, han puesto de manifiesto la necesidad de analizar el ámbito familiar tomando en cuenta la heterogeneidad y la diversidad de las dinámicas, las estructuras, las composiciones, el número de los integrantes, el nivel de las filiaciones, los arreglos de parentalidad (maternidad y paternidad) y de convivencia, así como las transformaciones orientadas hacia nuevas formas de vínculos interpersonales que se distancian del modelo tradicional donde los varones juegan los roles de jefes, sustentadores económicos exclusivos y referentes naturales ante el mundo exterior.

Las anteriores consideraciones cobran pertinencia a la luz del incremento de la jefatura femenina, los hogares unipersonales, las familias monoparentales, las familias inestables y reconstruidas, las uniones consensuales, los nacimientos extramatrimoniales y las madres solteras, así como el retraso en la entrada de las uniones matrimoniales, la baja de la fecundidad, la reducción del tamaño promedio de los hogares, y la disminución de los hogares multigeneracionales. De la misma forma, los autores previamente citados notan que las conclusiones de los investigadores latinoamericanos han mostrado 
la relevancia de tomar en cuenta la disimilitud de los intereses, la existencia de tensiones y conflictos latentes o intensos, y las relaciones jerárquicas asimétricas de poder entre los hombres y las mujeres en el hogar matrimonial. Asimismo, revelan la importancia de proceder a la deconstrucción y a la reconceptualización de las normas sociales entrelazadas con la organización de vida dentro de las unidades domésticas, como las imágenes sociales de las mujeres, y la división sexual de las actividades mercantiles y no mercantiles.

Como se puede entender, todos estos temas pueden ser abordados a la luz de la formulación teórico-conceptual de Catherine Hakim, como se ha hecho a través de las perspectivas teóricas de alcance individualista y de carácter convencional en los estudios sociodemográficos, por ejemplo, las de Gary Becker. Sin embargo, los matices explicativos no se construirían únicamente a partir de los puntos de encaje o de concordancia con la tesis de Hakim, sino también con los elementos de divergencia. Los puntos de divergencia y de convergencia darían la posibilidad de cuestionar, matizar, amplificar o reconstruir los interrogantes y las interpretaciones, para así generar nuevas evidencias empíricas acerca de la vivencia reproductiva y productiva de las mujeres. La influencia trascendental de las tesis de libertad, igualdad, equidad y de autonomía propuestas sobre todo por las corrientes feministas y las agencias internacionales que moldean y financian las agendas de las investigaciones y las políticas públicas sobre las familias, puede ser un argumento pertinente para intentar explicar la baja utilización de la tesis de las preferencias en la región latinoamericana y caribeña. Sin embargo, esta consideración tendría sus límites al tomar en cuenta la notoriedad de ciertas corrientes de pensamiento que comparten el paradigma individualista, racionalista o conservadurista de Hakim, como las de Becker y Giddens.

Habiendo esclarecido los puntos anteriores, cabe destacar que, con base en la premisa de acuerdo sobre que la población de las mujeres es heterogénea por lo que respecta a sus objetivos de vida, prioridades y aspiraciones familiares y profesionales, la tesis de Hakim plantea que sería un grave error concebir e implementar políticas públicas orientadas únicamente hacia un aspecto definido de la vida familiar y económica de las mujeres, tales como las que buscan: a) mejorar la participación de las mujeres sobre el mercado de trabajo; 
b) favorecer la igualdad de oportunidades entre los géneros sobre el mercado de trabajo; c) favorecer los roles simétricos entre hombres y mujeres en las actividades domésticas y extradomésticas; y d) mejorar la conciliación y reducir los conflictos de ambos espacios en la distribución del tiempo femenino (Hakim, 2011).

De acuerdo con el enfoque de Hakim, las políticas públicas familiares que promocionan el mejoramiento de la participación de las mujeres sobre el mercado de trabajo tendrían impactos muy bajos sobre las mujeres centradas en el hogar. De forma análoga, las legislaciones orientadas hacia la conciliación de las esferas de reproducción y laboral (mediante el financiamiento de acciones específicas, como servicios de guarderías, niñeras, asistencia domiciliaria, jardines de infantes accesibles, permisos parentales, permisos de maternidad, compensación económica, seguridad laboral) están condenadas al fracaso para las mujeres que desean asumir exclusivamente las responsabilidades domésticas o de la esfera productiva. Estos razonamientos son válidos también para las políticas públicas orientadas hacia la equiparación de oportunidades, recursos, tiempo, derechos y toma de decisión entre las mujeres y los hombres (discriminación positiva), que la autora evalúa como iniciativas infructuosas, contraproductivas y un desperdicio de fondos públicos.

Con base en estas aristas, las ideas de Hakim rechazan las políticas públicas dichas one-size-fits-all (modelo único para todos) y propone que las intervenciones tanto estatales como no estatales se diseñen y se adecuen de tal forma que sean neutras, no sesgadas, para evitar que tropiecen con las necesidades, los sueños, las aspiraciones, las preferencias, las prioridades, los anhelos, la subjetividad y los modelos ideales de familia propios de las mujeres. Estas medidas deben desprenderse de los prejuicios que se desarrollan en contra de las mujeres que desean quedarse en casa y ocuparse personalmente de la gestión de sus hogares y del cuidado de sus progenituras.

\section{Síntesis y consideraciones finales}

En el presente trabajo se ha intentado sintetizar, evaluar y esclarecer las reflexiones analíticas construidas alrededor de la tesis de las prefe- 
rencias que Catherine Hakim ha desarrollado en torno a la dicotomía familia y mercado de trabajo para la población de las mujeres. Siendo un enfoque fundamentalmente individualista, los postulados de Hakim tienen como telón de fondo la idea de que las características de la estructura social cobran un peso secundario en la elección que las mujeres realizan en cuanto a las esferas domésticas y ocupacionales. Hakim explica que, en las sociedades ricas, modernas y liberales, el peso de la estructura en el comportamiento de las mujeres está decreciendo notablemente. Las mujeres no están restringidas o forzadas a elegir estilos de vida, o tomar decisiones que no corresponden a la orientación que quieren dar a su vida ocupacional y familiar. Sus reflexiones toman como punto de partida la hipótesis de acuerdo con la cual la población femenina es heterogénea en lo que se trata de las preferencias, los gustos y la priorización de las esferas familiar y ocupacional. Esta diversidad influye destacadamente sobre el nivel de sensibilidad y la capacidad de respuesta de las mujeres ante las políticas públicas que buscan mejorar sus condiciones en una o ambas esferas.

Basándose en los postulados precedentes, la propuesta de Hakim presenta una tipología triple que trata de categorizar a las mujeres tomando en cuenta criterios conectados con sus preferencias e intereses entre la esfera familiar y el mercado de trabajo. La primera categoría reagrupa a las que están centradas en el hogar, en el sentido de que se dedican completamente a la gestión de las actividades domésticas, a menos que existan problemas económicos que pongan en riesgo la reproducción y el mantenimiento de la familia. La segunda categoría trata de las mujeres centradas en el mercado de trabajo (o en las actividades del sector público) en prejuicio de la vida familiar; éstas presentan un perfil completamente diferente a las de la primera categoría. La tercera categoría es la de las mujeres adaptativas, que son proporcionalmente más numerosas que aquellas centradas en el hogar y las centradas en el mercado de trabajo; las féminas que pertenecen al escenario adaptivo pueden ser más propensas a enfrentar conflictos y ambivalencias en lo que se trata de la gestión de las exigencias familiares y laborales, como en los papeles de madres y de mujeres económicamente activas. Se espera que la tasa de participación de las mujeres adaptativas sea más importante que la de aquellas centradas en el hogar, pero menos que las centradas en el trabajo. 
La autora ha demostrado que las mujeres centradas en el mercado de trabajo, o las que trabajan de tiempo completo, no son representativas de la mayoría femenina. De acuerdo con esta idea, plantea que los analistas deben ser más cuidadosos en el momento de interpretar los resultados de las mujeres sobre el mercado de trabajo, con fines de evitar explicaciones que pongan una atención desmedida sobre el patriarcado y los estereotipos de género. Hakim plantea que la búsqueda de equiparación en la capacidad y el tipo de inserción, como en las condiciones de trabajo tan esperadas y veneradas por corrientes importantes de pensamiento y de políticas públicas, no llegará mañana ni parece realista puesto que la población de las mujeres es más diversa en lo que se refiere al nivel de priorización de los trabajos reproductivos y productivos. Además, sólo una minoría de las mujeres quiere dedicarse complemente al mercado de trabajo, al contrario de las observaciones realizadas en la población de los hombres. Por esta razón, la autora piensa que es muy importante evitar las políticas públicas de tipo one-size-fits-all (modelo único para todos) para priorizar medidas adecuadas de tipo neutro, amplificado, diversificado, focalizado, eficaz y eficiente, que concuerde con los gustos, los valores, las aspiraciones y la subjetividad de las mujeres.

A la luz de las consideraciones precedentes y tomando en cuenta los bajos resultados obtenidos por las políticas públicas de protección social en la región latinoamericana y caribeña, se entiende la necesidad de explorar y adaptar nuevos referentes analíticos, como los propuestos por Hakim, en la medida que proporcionan nuevas categorías de interpretación capaces de incidir en el diseño, la gestión y la evaluación de las políticas públicas encaminadas a dar respuestas a una serie de necesidades y enfrentar los retos y los desafíos emergentes vinculados con las transformaciones y las reconfiguraciones de las formas de vivencia de las mujeres en el interior de los hogares matrimoniales y sobre el mercado de trabajo. Al respecto, entre las necesidades y los retos más importantes destacan la articulación y la conciliación armónica de las responsabilidades familiares y ocupacionales a través de las licencias de maternidad y de paternidad, las guarderías infantiles, la atención a personas adultas, la corresponsabilidad de las tareas vinculadas con la reproducción doméstica y el sostenimiento del hogar, las responsabilidades del cuidado y la re- 
distribución de sus costos. A estos problemas se añaden las vulnerabilidades socioeconómicas, las nuevas masculinidades y las nuevas feminidades.

\section{Bibliografía}

Acosta, F. (2003). La familia en los estudios de población en América Latina: estado del conocimiento y necesidades de investigación. Papeles de Población, 9(37), 1-51. http://www.scielo.org.mx/ scielo.php?script $=$ sci_arttext\&pid=S1405-74252003000300002 Arriagada, I. (2001a). Familias latinoamericanas. Diagnóstico y politicas públicas en los inicios del nuevo siglo. (Documento de trabajo, núm. 57). Serie Políticas Sociales. Santiago de Chile: Comisión Económica para América Latina y el Caribe. https:// repositorio.cepal.org/bitstream/handle/11362/6022/S01121052 es.pdf? sequence $=1$

Arriagada, I. (2001b). Políticas sociales, familia y trabajo en la América Latina del fin de siglo. (Documento de trabajo, núm. 21). Serie Políticas Sociales. Santiago de Chile: https://repositorio. cepal.org/bitstream/handle/11362/6221/S9700708_es.pdf? sequence $=1$ \&isAllowed $=\mathrm{y}$

Becker, G. (1965). A theory of allocation of time. The Economic Journal, 75(299), 493-517. https://www.jstor.org/stable/2228949? seq=1\#metadata_info_tab_contents

Becker, G. (1987). Tratado sobre la familia. Madrid: Alianza Universidad.

Blossfeld, H. y Hakim, C. (1997). Between equalization and marginalization. Women working part-time in Europe and the United States of America. Oxford: Oxford University Press.

Castel, R. (1994). La dynamique des processus de marginalisation: de la vulnérabilité à la désaffiliation. Cahiers de Recherche Sociologique, 22, 11-27. https://www.erudit.org/en/journals/ crs/1994-n22-crs1516985/1002206ar/

Castel, R. (1995). Les métamorphoses de la question sociale. París, Francia: Fayard. 
Castel, R. (2009). La montée des incertitudes. Travail, protections et statut de l'individu. París, Francia: Seuil.

Castel, R. (2010). El ascenso de las incertidumbres. Trabajo, protecciones, estatuto del individuo. Buenos Aires: Fondo de Cultura Económica.

Cartwright, S. (2004). Women's decisions about paid work and family life after childbirth: A Critique of the Hakim model. En S. Charlesworth y M. Fasteanu (eds.), Women and work (pp. 2740). Australia: RMIT Publishing.

Crompton, R. y Lyonette, C. (2005). The new gender essentialism-domestic and family choices and their relation to attitudes. The British Journal of Sociology, 56(4), 601-620. https://www. ncbi.nlm.nih.gov/pubmed/16309438

Crompton, R. Brockmann, M. y Lyonette, C. (2005). Attitudes, women's employment and domestic division and labor: A cross-national analysis in two waves. Work, Employment and Society, 19(2), 213-233. https://journals.sagepub.com/ doi/10.1177/0950017005053168

Del Valle, E. (2009). El empleo a tiempo parcial como herramienta para compatibilizar la flexibilidad laboral y la conciliación de la vida personal y laboral. España: Consejo Económico y Social.

De Oliveira, O. y Ariza, M. (1999). Trabajo, familia y condición femenina: una revisión de las principales perspectivas de análisis. Papeles de Población, 5(20), 89-127. https://www.redalyc.org/ pdf/112/Resumenes/Resumen_11202005_1.pdf

Doughney, J. y Leahy, M. (2006). Women, work and preference formation: A critique of Catherine Hakim's preference theory. Journal of Business Systems, Governance and Ethics, 1(1), 37-48. https://jbsge.vu.edu.au/index.php/jbsge/article/view/79/130

Duncan, S. (2006). Mothers' work-life balance: Individualized preferences or cultural construction? En D. Perrons, C. Fagan, L. McDowell, K. Ray y K. Ward (eds.), Gender divisions and working time in the new economy (pp. 127-147) Cheltenham: Edward Elgar Publishing.

Duncan, S. e Irwin, S. (2004). The social patterning of values and rationalities. Social Policy and Society, 3(4), 391-399. http:// eprints.whiterose.ac.uk/1295/ 
García, B. y De Oliveira, O. (1994). Trabajo femenino y vida familiar en México. Ciudad de México, El Colegio de México, A.C. García, B. y De Oliveira, O. (2007). Trabajo extradoméstico y relaciones de género: una nueva mirada. En M. A. Gutiérrez (comp.), Género, familias y trabajo: rupturas y continuidades. Desafios para la investigación politica (pp. 49-87). Buenos Aires: Consejo Latinoamericano de Ciencias Sociales.

García, B. y Rojas, O. (2002). Los hogares latinoamericanos durante la segunda mitad del siglo XX: una perspectiva sociodemográfica. Estudios Demográficos y Urbanos, 17(2), 261-288. https:// estudiosdemograficosyurbanos.colmex.mx/index.php/edu/ article/view/1139/1132

Gash, V. (2008). Preference or constraint? Part-time workers' transitions in Denmark, France and the United Kingdom. Work Employment Society, 22(4), 655-674. https://journals.sagepub.com/ doi/10.1177/0950017008096741

Ginn, J., Arber, S., Brannen, J., Dale, A., Dex, S., Elias, P., Moss, P., Pahl, J., Roberts, C. y Rubery, J. (1996). Feminist fallacies: A reply to Hakim on women's employment. The British Journal of Sociology, 47(1), 167-174. https://www.ncbi.nlm.nih.gov/ pubmed/8680791

González de la Rocha, M. (coord.) (1999). Divergencias del modelo tradicional: hogares de jefatura femenina en América Latina. Ciudad de México: CIESAS / SEP/ Conacyt / Plaza y Valdés.

Hakim, C. (1995). Five feminist myths about women's employment. The British Journal of Sociology, 46(3), 429-455. http://eprints. lse.ac.uk/25032/

Hakim, C. (2000). Work-lifestyle choices in the 21st century. Preference theory. Nueva York: Oxford University Press.

Hakim, C. (2002). Lifestyle preferences as determinants of women's differentiated labor market careers. Work and Occupations, 29(4), 428-459. https://journals.sagepub.com/doi/10.1177/ 0730888402029004003

Hakim, C. (2003a). A new approach to explaining fertility patterns: Preference theory. Population and Development Review, 29(3), 349-374. https://www.jstor.org/stable/3115278?seq=1\#metadata info_tab_contents 
Hakim, C. (2003b). Public morality versus personal choice: The failure of social attitude surveys. The British Journal of Sociology, 54(3), 339-345. https://www.ncbi.nlm.nih.gov/pubmed/ 14514462

Hakim, C. (2004). Key issues in women's work: Female diversity and the polarization of women's employment. Londres: Glass House Press.

Hakim, C. (2006). Women, careers, and work-life preferences. British Journal of Guidance and Counselling, 34(3), 279-294. https:// www.tandfonline.com/doi/full/10.1080/03069880600769118

Hakim, C. (2007). Dancing with the devil? Essentialism and other feminist heresies. The British Journal of Sociology, 58(1), 123-132. https://onlinelibrary.wiley.com/doi/full/10.1111/j.1468-4446. 2007.00142.x

Hakim, C. (2008a). Diversity in tastes, values, and preferences: Comment on Jonung and Ståhlberg. Econ Journal Watch, 5(2), 204-218.https://econjwatch.org/articles/diversity-in-tastes-valuesand-preferences-comment-on-jonung-and-stahlberg

Hakim, C. (2008b). Is gender equality legislation becoming counter-productive? Public Policy Research, 15(3), 133-136. https:// onlinelibrary.wiley.com/doi/abs/10.1111/j.1744-540X.2008. 00528.x

Hakim, C. (2011). Feminist myths and magic medicine: The flawed thinking behind calls for further equality legislation. Londres: Centre for Policy Studies.

Himmelweit, S. y Sigala, M. (2004). Choice and the relationship between identities and behaviour for mothers with pre-school children: Some implications for policy from a UK study. Journal of Social Policy, 33(3), 455-478. https://pdfs.semanticscholar. org/11ea/e0f5caa19e1d419b161a40ed0bbdcb675aaf.pdf

Humphries, J. y Rubery, J. (1994). La autonomía relativa de la reproducción social. En C. Bordería, C. Carrasco y C. Alemany (comps.), Las mujeres y el trabajo: rupturas conceptuales (pp. 393-424). Barcelona: Icaria

Kangas, O. y Rostgaard, T. (2007). Preferences or institutions? Work-family life opportunities in seven European countries. Jour- 
nal of European Social Policy, 17(3), 240-256. https://journals. sagepub.com/doi/10.1177/0958928707078367

Man Yee, K. (2007). Work orientation and wives' employment careers: An evaluation of Hakim's preference theory. Work and Occupations, 34(4), 430-462. https://journals.sagepub.com/ doi $/ 10.1177 / 0730888407307200$

Mara, Y. (2013). Choice or constraint? Women's weekly working hours in comparative perspective. Sociologia. Problemas e Práticas, 72, 9-30. https://journals.openedition.org/spp/1156

McRae, S. (2003). Constraints and choices in mother's employment careers: A considerations of Hakim's preference theory. The British Journal of Sociology, 54(3), 317-338. https://www.ncbi.nlm. nih.gov/pubmed/14514461

Méda, D. (1995). Le travail: Une valeur en voie de disparition. París, Francia: Aubier.

Navarro, A. (2006). Modelos ideales de familia en la sociedad española. Revista Internacional de Sociología, 64(43) 119-138. http://www.acuedi.org/ddata/F10395.pdf

Perrons, D., McDowell, L., Fagan, C., Ray, K. y Ward, K. (2007). Gender, social class and work-life balance in the new economy. En C. Rosemary, S. Lewis y C. Lyonette (eds.), Women, men, work and family in Europe (pp. 133-151). Basingstoke: Palgrave Macmillan.

Phillips, A. (2004). Defending equality of outcome. The Journal of Political Philosophy, 12(1), 1-19. https://onlinelibrary.wiley. com/doi/epdf/10.1111/j.1467-9760.2004.00188.x

Philp, B. y Wheatley, D. (2010). The scarcity and the dual career households: Competing perspectives. (Discussion Papers in Economics, núm. 2010/6). Gran Bretaña: Nottingham Trent University. https://www.ntu.ac.uk/_data/assets/pdf_file/0016/310228/ The-scarcity-and-the-dual-career-household.pdf

Prieto, C. (2000). Trabajo y orden social: de la nada a la sociedad de empleo (y su crisis). Revista Política y Sociedad, 34, 19-32. https://dialnet.unirioja.es/servlet/articulo?codigo=154599

Prieto, C. (2007). De la perfecta casada a la conciliación de la vida familiar y laboral o la querelle des sexes en la modernidad espa- 
ñola. En C. Prieto (ed.), Trabajo, género y tiempo social (pp. 4963). Madrid: Universidad Complutense de Madrid.

Sánchez Mira, N. (2016). La división social y sexual del trabajo en transformación. Un análisis de clase en un contexto de crisis. (Doctorado en Sociología, Departamento de Sociología, Facultad de Ciencias Políticas y Sociología, Universidad Autónoma de Barcelona). https://ddd.uab.cat/pub/tesis/2016/ hdl_10803_399581/nsm1de1.pdf

Schleutker, E. (2013). Fertility, family policy and welfare regimen. Comparative Population Studies, 39(1), 123-156. http:// www.comparativepopulationstudies.de/index.php/CPoS/article/ view/129

Tobio, C. (2005). Madres que trabajan. Dilemas y estrategias. Valencia: Universitat de València.

Tomlinson, J. (2006). Women's work-life balance trajectories in the UK: Reformulating choice and constraint in transitions through part-time work across the life-course. British Journal of Guidance and Counselling, 34(3), 365-382. https://www.tandfonline. com/doi/full/10.1080/03069880600769555

Vega-Robles, I. (2007). Relaciones de equidad entre hombres y mujeres. Análisis crítico del entorno familiar. Actualidades en Psicología, 21(108), 59-78. https://revistas.ucr.ac.cr/index.php/ actualidades/article/view/30

Walter, S. (2005). Making the best of a bad job? Female part-timers' orientations and attitudes to work. Gender Work and Organization, 12(3), 193-216. https://onlinelibrary.wiley.com/doi/ full/10.1111/j.1468-0432.2005.00270.x

Yerkes, M. (2013). Choice or constraint? Women's weekly working hours in comparative perspective. Sociologia, Problemas e Práticas, 72, 9-30. http://journals.openedition.org/spp/1156

\section{Acerca del autor}

Mickens Mathieu es doctor en Estudios de Población por El Colegio de México, A.C.; es maestro en Población y Desarrollo, y licenciado en Economía y Desarrollo Rural, ambos por la Universidad 
Estatal de Haití. Sus áreas de interés son: movimientos migratorios en el espacio latinoamericano y caribeño; desigualdades de género y etnonacionales; proceso de integración de los inmigrantes en el mercado laboral de los países de acogida; focalización geográfica de las poblaciones que viven en condiciones de pobreza. Actualmente es profesor de la Facultad de Ciencias Humanas de la Universidad Estatal de Haití; también funge como consultor de organismos internacionales, como el Banco Mundial y el Fondo de Población de las Naciones Unidas. ORCID: https://orcid.org/0000-0001-5520-6477

Entre sus publicaciones se encuentran:

Mickens, M. (2019). L'espace familial et l'offre de travail des femmes: une synthèse des contributions théoriques sur le sujet. Haïti Perspectives, 7(56), 45-57. https://www.researchgate.net/publication/340936021_L'espace_familial_et_l'offre_de_travail_ des_femmes_une_synthese_des_contributions_theoriques_sur_ le_sujet

Mickens, M. (2019). La participation d'Haïti à la quatrième réunion de la CEPAL sur le Consensus de Montevideo. Reporte de Investigación, Association Nationale des Specialistes en Population et Dévéloppement. https://hal.archives-ouvertes.fr/hal-02544090/ document

Mickens, M. (2019). Etat des lieux du système d'enregistrement des naissances en Haïti. Documento de Trabajo. Universidad Estatal de Haití / Association Nationale des Spécialistes en Population et Développement. https://hal.archives-ouvertes.fr/hal-02544096/ document

Recepción: 24 de abril de 2018. Aceptación: 9 de enero de 2020. 
